\title{
Variation of quasiconformal mappings on lines
}

\author{
by \\ LeOnid V. Kovalev and Jani Onninen (Syracuse, NY)
}

\begin{abstract}
We obtain improved regularity of homeomorphic solutions of the reduced Beltrami equation, as compared to the standard Beltrami equation. Such an improvement is not possible in terms of Hölder or Sobolev regularity; instead, our results concern the generalized variation of restrictions to lines. Specifically, we prove that the restriction to any line segment has finite $p$-variation for all $p>1$ but not necessarily for $p=1$.
\end{abstract}

1. Introduction. A key property of Sobolev functions in Euclidean spaces is their absolute continuity on almost every line parallel to the coordinate axes. The restrictions to arbitrary lines need not be even bounded for functions in Sobolev spaces $W^{1, s}, 1 \leq s \leq n$. However, for $s>n$ the restriction of a Sobolev function to any line has finite $p$-variation with $p=s /(s-n+1)$ (see Remark 2.1). Here we refer to the generalized variation $[19,23,24]$, which is defined as follows.

For distinct points $a, b \in \mathbb{R}^{n}$ we write $[a, b]=\{(1-t) a+t b: 0 \leq t \leq 1\}$ and call $[a, b]$ the line segment with endpoints $a$ and $b$. Any partition $0=t_{0}<$ $t_{1}<\cdots<t_{N}=1$ induces a partition of $[a, b]$ by the rule $a_{j}=a+t_{j}(b-a)$, $j=0, \ldots, N$.

Definition 1.1. Let $\phi:[0, \infty) \rightarrow[0, \infty)$ be a convex strictly increasing function such that $\phi(0)=0$. A mapping $f$ from a line segment $[a, b] \subset \mathbb{R}^{n}$ into $\mathbb{R}^{m}$ has finite $\phi$-variation on $[a, b]$ if

$$
\operatorname{var}_{[a, b]}(f ; \phi):=\sup \sum_{j=1}^{N} \phi\left(\left|f\left(a_{j}\right)-f\left(a_{j-1}\right)\right|\right)<\infty,
$$

where the supremum is taken over all partitions $\left(a_{j}\right)_{j=0}^{N}$ of $[a, b]$ and over all $N \geq 1$.

2010 Mathematics Subject Classification: Primary 30C62; Secondary 26A45, 30C65, 26B30.

Key words and phrases: bounded variation, generalized variation, quasiconformal mappings, quaternions. 
If $f$ is a mapping from a domain $\Omega \subset \mathbb{R}^{n}$ into $\mathbb{R}^{m}$, then we say that $f$ has finite $\phi$-variation on lines if its restriction to any compact line segment contained in $\Omega$ has finite $\phi$-variation. When $\phi(t)=t^{p}$, we speak of $p$-variation (or simply variation if $p=1$ ) and write $\operatorname{var}_{[a, b]}(f ; p)$.

We are primarily interested in the variation of quasiconformal mappings $f: \mathbb{R}^{n} \rightarrow \mathbb{R}^{n}$, where $n \geq 2$. Recall that a sense-preserving homeomorphism $f: \mathbb{R}^{n} \rightarrow \mathbb{R}^{n}$ is said to be quasiconformal if there exists $H<\infty$ such that

$$
\limsup _{r \rightarrow 0} \frac{\max _{|x-a|=r}|f(x)-f(a)|}{\min _{y-a \mid=r}|f(y)-f(a)|} \leq H \quad \text { for every } a \in \mathbb{R}^{n} .
$$

It is a well-known result of Gehring [9] that such mappings are absolutely continuous on almost every line (ACL). This was recently extended to Ahlfors regular metric spaces by Balogh, Koskela and Rogovin [4]. The ACL property makes it possible to give an analytic definition of quasiconformal mappings.

Definition 1.2. A homeomorphism $f \in W_{\text {loc }}^{1, n}\left(\mathbb{R}^{n} ; \mathbb{R}^{n}\right)$ is $K$-quasiconformal, $1 \leq K<\infty$, if it satisfies the distortion inequality

$$
\|D f(x)\|^{n} \leq K J(x, f) \quad \text { a.e. }
$$

Here $\|D f(x)\|$ stands for the norm of the differential matrix and $J(x, f)$ for the Jacobian determinant. A mapping $f \in W_{\text {loc }}^{1, n}\left(\mathbb{R}^{n} ; \mathbb{R}^{n}\right.$ ) (not necessarily a homeomorphism) satisfying (1.2) is called $K$-quasiregular [20, 21].

Our study of the variation of quasiconformal mappings on lines grew out of [14] where it was proved that the ordinary differential equation $\dot{x}=f(x)$ has unique local solutions outside of $f^{-1}(0)$ provided that $f$ is quasiconformal and has bounded variation on $C^{1}$-smooth curves. By Gehring's theorem [10] quasiconformal mappings are locally in $W^{1, s}$ for some $s>n$ and therefore have finite $p$-variation on lines for some $p<n$. (In fact, any homeomorphism of class $W^{1, n}$ has finite $n$-variation on lines [18, Thm. 4.3].) In the opposite direction, a theorem of Bishop [5, Thm. 1.1] implies that for any $p<n$ there is a quasiconformal mapping $f: \mathbb{R}^{n} \rightarrow \mathbb{R}^{n}$ such that the image of some line segment under $f$ has Hausdorff dimension greater than $p$. Clearly, such an $f$ has infinite $p$-variation on this segment.

Interestingly, some classes of quasiconformal mappings exhibit much higher regularity along lines and smooth curves than their Sobolev or Hölder regularity would suggest.

Definition 1.3. A mapping $f: \mathbb{R}^{n} \rightarrow \mathbb{R}^{n}$ is called $\delta$-monotone, $0<\delta$ $\leq 1$, if for every $a, b \in \mathbb{R}^{n}$,

$$
\langle f(a)-f(b), a-b\rangle \geq \delta|f(a)-f(b)||a-b| .
$$

Any nonconstant $\delta$-monotone mapping is quasiconformal [15, Cor. 7]. For example, the radial stretch $f(x)=|x|^{\alpha-1} x$, where $\alpha>0$, is $\delta$-monotone 
for some $\delta=\delta(\alpha)$. This mapping is locally Hölder continuous with exponent $\min \{\alpha, 1\}$, which can be arbitrarily close to 0 . This shows that $\delta$-monotone mappings are no more regular on the Hölder and Sobolev scales than general quasiconformal mappings. However, they have bounded variation on $C^{1}$ smooth curves (see [2, Thm. 3.11.7] and [14, Thm. 1.10]). In particular,

$$
\underset{[a, b]}{\operatorname{var}}(f ; 1)<\infty \quad \text { for } a, b \in \mathbb{R}^{n} .
$$

When $n=2$, we often identify $\mathbb{R}^{n}$ with $\mathbb{C}$ and use the complex derivatives $f_{z}$ and $f_{\bar{z}}$. Then the inequality (1.2) reads

$$
\left|f_{\bar{z}}\right| \leq k\left|f_{z}\right| \quad \text { a.e., } \quad \text { where } k=\frac{K-1}{K+1} .
$$

A $\delta$-monotone mapping $f: \mathbb{C} \rightarrow \mathbb{C}$ satisfies the stronger, reduced distortion inequality

$$
\left|f_{\bar{z}}\right| \leq k \operatorname{Re} f_{z} \quad \text { a.e. in } \mathbb{C},
$$

for some constant $0<k<1$ ([2, Thm. 3.11.6]). The converse is false: for instance, $f(z)=i z$ satisfies (1.6) but is not $\delta$-monotone.

Definition 1.4. A homeomorphism $f \in W_{\text {loc }}^{1,2}(\mathbb{C} ; \mathbb{C})$ is called reduced quasiconformal if it satisfies (1.6).

By Corollary 1.5 in [13] any nonconstant solution of (1.6) in the class $W_{\text {loc }}^{1,2}(\mathbb{C} ; \mathbb{C})$ is a homeomorphism. This is false for mappings defined in a domain $\Omega \subset \mathbb{C}$ (see [13, Example 5.2]).

Inequality (1.6) implies that $f$ is a solution of the reduced Beltrami equation

$$
f_{\bar{z}}=\lambda(z) \operatorname{Re} f_{z} \text { a.e. in } \mathbb{C}
$$

where $|\lambda(z)| \leq k$. Conversely, it is shown in [2, Thm. 6.3.2] that any homeomorphic solution of (1.7) has constant sign of $\operatorname{Re} f_{z}$. Therefore such solutions satisfy (1.6) up to a change of sign.

Unlike quasiconformality, the properties (1.3) and (1.6) are preserved under addition. Both of these classes arise naturally in the theory of elliptic partial differential equations $[1,2,3]$. Our first result shows that (1.4) cannot be extended to reduced quasiconformal mappings.

THEOREM 1.5. For every $k \in(0,1)$ there exists a reduced quasiconformal mapping $f: \mathbb{C} \rightarrow \mathbb{C}$ that satisfies (1.6) but does not have bounded variation on any nontrivial interval $[a, b] \subset \mathbb{R}$. Furthermore, $f$ can be chosen so that $f_{\bar{z}}, \operatorname{Re} f_{z} \in L^{\infty}(\mathbb{C})$ and $\operatorname{Re} f(x)=x$ for all $x \in \mathbb{R}$.

In other words, $f$ in Theorem 1.5 maps $\mathbb{R}$ into a curve that is nowhere locally rectifiable. Although such examples of (nonreduced) quasiconformal mappings were known for a long time [22], our mapping $f$ seems to be the first one given by an explicit analytic expression (see (4.1)). The additive 
property of reduced quasiconformal mappings allows us to derive the following result from Theorem 1.5.

Corollary 1.6. For any countable family $\left\{L_{j}: j=1,2, \ldots\right\}$ of parallel lines in $\mathbb{C}$ there exists a reduced quasiconformal mapping $f: \mathbb{C} \rightarrow \mathbb{C}$ such that any nontrivial subarc of $f\left(L_{j}\right)$ is unrectifiable for every $j$.

Corollary 1.6 exhibits a quasiconformal mapping with irregular behavior on a relatively large set. The authors of [6] asked (Question 4.4) whether for any set $E \subset \mathbb{C}$ of planar measure zero there is a quasiconformal mapping $f: \mathbb{C} \rightarrow \mathbb{C}$ such that the volume derivative $\lim _{r \rightarrow 0}|f(B(x, r))| /|B(x, r)|$ is infinite at every point of $E$. While the singular behavior of $f$ in Corollary 1.6 is of different nature, the additive property of reduced quasiconformal mappings can be potentially useful in creating mappings with a large set of infinite volume derivative.

Our second main result shows that, Theorem 1.5 nonwithstanding, reduced quasiconformal mappings are much more regular on lines than general quasiconformal mappings. In particular, they have finite $p$-variation for any $p>1$.

TheOREM 1.7. Let $f: \mathbb{C} \rightarrow \mathbb{C}$ be a reduced quasiconformal mapping. Then for any $q>1$ the mapping $f$ has finite $\phi$-variation on lines with

$$
\phi(t)=\frac{t}{(\log (e+1 / t))^{q}} \quad \text { if } t>0 \quad \text { and } \quad \phi(0)=0 .
$$

The conclusion of Theorem 1.7 is false for $0 \leq q<1 / 2$ (see Remark 4.1). The gap between exponents $1 / 2$ and 1 remains open. Although in this paper we chose to focus on mappings defined on the entire plane $\mathbb{C}$, the proof of Theorem 1.7 can be adapted to reduced quasiconformal homeomorphisms $f: \Omega \rightarrow \mathbb{C}$, where $\Omega$ is a convex domain in $\mathbb{C}$. See Remark 3.2.

QUESTION 1.8. What is the smallest value of $q$ for which the conclusion of Theorem 1.7 holds?

Since $\delta$-monotone mappings exist in any dimension $n \geq 2$, one may ask whether it is possible to extend the definition of reduced quasiconformal mappings to higher dimensions. This question is addressed in Section 5, where we use quaternions to define reduced quasiconformal mappings in four dimensions, and extend Theorem 1.7 to them.

QUESTION 1.9. Is there a natural analogue of reduced quasiconformal mappings in dimensions other than 2 and 4 ?

2. Preliminaries. In this section we first estimate the $p$-variation of Sobolev functions on lines. Although this result is probably known, we give 
a proof for the sake of completeness. Later in the section we define quasisymmetric and monotone mappings and introduce some relevant notation. In this paper $\Omega$ stands for a domain in $\mathbb{R}^{n}$.

Proposition 2.1. Let $u \in W^{1, s}(\Omega), s>n$. Then the restriction of $u$ to any closed line segment $I \subset \Omega$ has finite p-variation with $p=s /(s-n+1)$.

The Morrey-Sobolev embedding theorem states that $W^{1, s}(\Omega) \subset C_{\mathrm{loc}}^{\alpha}(\Omega)$ with $\alpha=1-n / s$. Clearly, any function $u \in C_{\mathrm{loc}}^{\alpha}(\Omega)$ has finite $p$-variation on lines with $p=1 / \alpha=s /(s-n)$. However, Proposition 2.1 gives a better value of $p$. Its proof requires the following lemma.

LEMMA 2.2. Let I be a line segment partitioned into smaller segments $I_{m}, m=1, \ldots, M$. For any mapping $f: I \rightarrow \mathbb{R}^{n}$ we have

$$
\operatorname{var}_{I}(f ; \phi) \leq \sum_{m=1}^{M} \operatorname{var}(f ; \varphi)+(M-1) \phi(\underset{I}{\operatorname{osc} f}) .
$$

Proof. Fix a partition $\left(a_{j}\right)_{j=0}^{N}$ of $I$. We divide the set of indices as follows. $E=\left\{j=1, \ldots, N:\left[a_{j-1}, a_{j}\right] \subset I_{m}\right.$ for some $\left.m\right\}, \quad F=\{1, \ldots, N\} \backslash E$. Then

$$
\sum_{j \in E} \phi\left(\left|f\left(a_{j}\right)-f\left(a_{j-1}\right)\right|\right) \leq \sum_{m=1}^{M} \operatorname{var}_{I_{m}}(f ; \varphi)
$$

and

$$
\sum_{j \in F} \phi\left(\left|f\left(a_{j}\right)-f\left(a_{j-1}\right)\right|\right) \leq(M-1) \phi(\underset{I}{\operatorname{osc}} f) .
$$

Proof of Proposition 2.1. Dividing I into subintervals and using Lemma 2.2 we may reduce our task to the case

$$
\operatorname{diam} I<\operatorname{dist}(I, \partial \Omega) \text {. }
$$

Let $\left(a_{j}\right)_{j=0}^{N}$ be a partition of $I$. For $j=1, \ldots, N$ let $\bar{B}_{j}$ be the closed ball with segment $\left[a_{j-1}, a_{j}\right]$ as a diameter. Morrey's inequality [7, p. 143] yields

$$
\overline{\mathrm{OSC}}_{j} u \leq C\left|a_{j}-a_{j-1}\right|^{1-n / s}\left(\int_{B_{j}}|\nabla u(x)|^{s} d x\right)^{1 / s} .
$$

Raising to the power $p$ and noticing that $(1-n / s) p=1-p / s$ we arrive at

$$
\left(\frac{\operatorname{osc} u}{\bar{B}_{j}}\right)^{p} \leq C\left|a_{j}-a_{j-1}\right|^{1-p / s}\left(\int_{B_{j}}|\nabla u(x)|^{s} d x\right)^{p / s} .
$$

Summing over $j$ and applying Hölder's inequality we obtain

$$
\sum_{j=1}^{N}\left(\operatorname{osc}_{j} u\right)^{p} \leq C\left(\sum_{j=1}^{N}\left|a_{j}-a_{j-1}\right|\right)^{1-p / s}\left(\sum_{j=1}^{N} \int_{B_{j}}|\nabla u(x)|^{s} d x\right)^{p / s} .
$$


Therefore

as desired.

$$
\underset{I}{\operatorname{var}}(u ; p) \leq C(\operatorname{diam} I)^{1-p / s}\left(\int_{\Omega}|\nabla u(x)|^{s} d x\right)^{p / s}
$$

Definition 2.3. Let $\eta:[0, \infty) \rightarrow[0, \infty)$ be a homeomorphism. An injective mapping $f: \mathbb{R}^{n} \rightarrow \mathbb{R}^{n}$ is $\eta$-quasisymmetric if

$$
\frac{|f(c)-f(a)|}{|f(b)-f(a)|} \leq \eta\left(\frac{|c-a|}{|b-a|}\right)
$$

for any distinct points $a, b, c \in \mathbb{R}^{n}$. The function $\eta$ is called a modulus of quasisymmetry of $f$.

It is well-known that a mapping $f: \mathbb{R}^{n} \rightarrow \mathbb{R}^{n}$ is quasiconformal if and only if it is sense-preserving and quasisymmetric [11].

Given a mapping $f: \mathbb{R}^{n} \rightarrow \mathbb{R}^{n}$ we define the modulus of monotonicity $\Delta_{f}: \mathbb{R}^{n} \times \mathbb{R}^{n} \rightarrow \mathbb{R}$ by the rule

$$
\Delta_{f}(a, b)= \begin{cases}\left\langle f(a)-f(b), \frac{a-b}{|a-b|}\right\rangle & \text { if } a \neq b, \\ 0 & \text { if } a=b .\end{cases}
$$

Clearly $\left|\Delta_{f}(a, b)\right| \leq|f(a)-f(b)|$. By definition, $f$ is a monotone mapping if $\Delta_{f}(a, b) \geq 0$ for all $a, b \in \mathbb{R}^{n}$, and is strictly monotone if $\Delta_{f}(a, b)>0$ unless $a=b$. Any reduced quasiconformal is monotone by (1.9) in [14]. Also, $f$ is $\delta$-monotone if and only if $\Delta_{f}(a, b) \geq \delta|f(a)-f(b)|$ for all $a, b \in \mathbb{R}^{n}$. When $n=2$, the modulus of monotonicity can be expressed in complex notation:

$$
\Delta_{f}(a, b)=\operatorname{Re}\left(\frac{f(a)-f(b)}{a-b}\right)|a-b| .
$$

Let us observe that any reduced quasiconformal mapping $f: \mathbb{C} \rightarrow \mathbb{C}$ is monotone. Indeed, by the ACL property of $f$ it suffices to prove $\Delta_{f}(a, b) \geq 0$ when $f$ is absolutely continuous on the line segment $[a, b]$. In this case

$$
\frac{f(a)-f(b)}{a-b}=\int_{0}^{1}\left(f_{z}(b+t(a-b))+f_{\bar{z}}(b+t(a-b)) \frac{\overline{a-b}}{a-b}\right) d t .
$$

Taking the real part of both sides and using (1.6), we obtain $\Delta_{f}(a, b) \geq 0$. Note that this argument is also valid when the domain of definition of $f$ is a convex open set.

3. Generalized variation on lines: Proof of Theorem 1.7. We will obtain Theorem 1.7 as a consequence of the following result.

THEOREM 3.1. Let $f: \Omega \rightarrow \mathbb{R}^{n}$ be a mapping and suppose that there is a homeomorphism $\eta:[0, \infty) \rightarrow[0, \infty)$ such that

$$
|f(c)-f(a)| \leq \frac{|c-a|}{|b-a|}|f(b)-f(a)|+\eta\left(\frac{|c-a|}{|b-a|}\right) \Delta_{f}(a, b)
$$


for any distinct points $a, b, c \in \Omega$. Then for any $q>1$ the mapping $f$ has finite $\phi$-variation on lines with $\phi$ as in (1.8).

Before proving Theorem 3.1 we derive Theorem 1.7 from it.

Proof of Theorem 1.7. Let $f: \mathbb{C} \rightarrow \mathbb{C}$ be a reduced quasiconformal mapping. We may assume that $f$ is nonlinear. For any $\lambda \in \mathbb{R}$ the mapping $f^{\lambda}(z)=f(z)+i \lambda z$ also satisfies the reduced distortion inequality (1.6) with the same constant $k$ as $f$. By [13, Cor. 1.5], $f^{\lambda}$ is a homeomorphism. Therefore, $f^{\lambda}$ is $K$-quasiconformal with $K$ independent of $\lambda$. Since quasiconformality implies quasisymmetry in $\mathbb{C}[11$, Thm. 11.14], there is a homeomorphism $\eta:[0, \infty) \rightarrow[0, \infty)$ such that $f^{\lambda}$ is $\eta$-quasisymmetric in $\mathbb{C}$ for all $\lambda \in \mathbb{R}$. Given distinct points $a, b, c \in \mathbb{R}$, let $\lambda=-\operatorname{Im} \frac{f(b)-f(a)}{b-a}$, so that $\left|f^{\lambda}(b)-f^{\lambda}(a)\right|=\Delta_{f}(a, b)$. Since $f^{\lambda}$ is $\eta$-quasisymmetric, we have

$$
\left|f^{\lambda}(c)-f^{\lambda}(a)\right| \leq \eta\left(\frac{|c-a|}{|b-a|}\right)\left|f^{\lambda}(b)-f^{\lambda}(a)\right|,
$$

from which (3.1) follows by means of the triangle inequality. It remains to apply Theorem 3.1 to $f$.

REMARK 3.2. In the preceding proof one can establish the injectivity of $f^{\lambda}$ without using [13, Cor. 1.5]. As observed at the end of Section $2, f$ is monotone. Given $\epsilon>0$, let $g(z)=f^{\lambda}(z)+\epsilon z$. Note that $g$ satisfies (1.6) with the same constant $k$ as $f$. Since $\Delta_{g}(a, b)=\Delta_{f}(a, b)+\epsilon|a-b|$, the mapping $g$ is strictly monotone, hence injective. Letting $\epsilon \rightarrow 0$, we conclude that $f^{\lambda}$ is either quasiconformal or constant [17, II 5.3]. The latter case does not occur since $f$ is not linear.

Our proof of Theorem 3.1 is based on two lemmas.

Lemma 3.3. Let $f: \Omega \rightarrow \mathbb{R}^{n}$ be as in Theorem 3.1. Given distinct points $a, b \in \Omega$ and a partition $0=t_{0}<t_{1}<\cdots<t_{N}=1$, let $a_{j}=a+t_{j}(b-a)$, $j=0, \ldots, N$. If $a_{j} \in \Omega$ for all $j$, then

$$
\sum_{j=1}^{N}\left|f\left(a_{j}\right)-f\left(a_{j-1}\right)\right| \leq C \log (N+1)|f(a)-f(b)|
$$

where the constant $C$ depends only on $\eta$ in (3.1).

Proof. It suffices to prove (3.2) for $N=2^{m}$. For $j=1, \ldots, 2^{m-1}$ we apply (3.1) to the points $a_{2 j}, a_{2 j-1}, a_{2 j-2}$ and find that $\left|f\left(a_{2 j}\right)-f\left(a_{2 j-1}\right)\right| \leq \frac{\left|a_{2 j}-a_{2 j-1}\right|}{\left|a_{2 j}-a_{2 j-2}\right|}\left|f\left(a_{2 j}\right)-f\left(a_{2 j-2}\right)\right|+\eta(1) \Delta_{f}\left(a_{2 j}, a_{2 j-2}\right)$ 
and

$$
\begin{aligned}
\mid f\left(a_{2 j-1}\right)- & f\left(a_{2 j-2}\right) \mid \\
& \leq \frac{\left|a_{2 j-1}-a_{2 j-2}\right|}{\left|a_{2 j}-a_{2 j-2}\right|}\left|f\left(a_{2 j}\right)-f\left(a_{2 j-2}\right)\right|+\eta(1) \Delta_{f}\left(a_{2 j}, a_{2 j-2}\right) .
\end{aligned}
$$

Adding these inequalities we obtain

$$
\begin{aligned}
\left|f\left(a_{2 j}\right)-f\left(a_{2 j-1}\right)\right| & +\left|f\left(a_{2 j-1}\right)-f\left(a_{2 j-2}\right)\right| \\
& \leq\left|f\left(a_{2 j}\right)-f\left(a_{2 j-2}\right)\right|+2 \eta(1) \Delta_{f}\left(a_{2 j}, a_{2 j-2}\right) .
\end{aligned}
$$

Observe that $\Delta_{f}(x, y)+\Delta_{f}(y, z)=\Delta_{f}(x, z)$ for all $y \in[x, z]$. Therefore, summation over $j=1, \ldots, 2^{m-1}$ yields

$$
\sum_{j=1}^{2^{m}}\left|f\left(a_{j}\right)-f\left(a_{j-1}\right)\right| \leq \sum_{j=1}^{2^{m-1}}\left|f\left(a_{2 j}\right)-f\left(a_{2 j-2}\right)\right|+2 \eta(1) \Delta_{f}(a, b) .
$$

Notice that the sum on the right hand side involves only even indices. Next we apply (3.3) to the partition $a_{0}, a_{2}, \ldots, a_{2^{m}}$ to further reduce the number of points. After $m$ steps we arrive at

$\sum_{j=1}^{2^{m}}\left|f\left(a_{j}\right)-f\left(a_{j-1}\right)\right| \leq|f(a)-f(b)|+2 m \eta(1) \Delta_{f}(a, b) \leq(2 m+1)|f(a)-f(b)|$.

This completes the proof of (3.2).

The following lemma will allow us to derive the conclusion of Theorem 3.1 from the growth estimate (3.2).

LEMMA 3.4. Suppose that $d_{1} \geq \cdots \geq d_{N}$ are positive numbers such that for $j=1, \ldots, N$ the partial sum $s_{j}:=d_{1}+\cdots+d_{j}$ is bounded by $C \log (j+1)$, where $C$ is a constant. Let $q>1$ and define $\phi$ by (1.8). Then

$$
\sum_{j=1}^{N} \phi\left(d_{j}\right) \leq C^{\prime}
$$

where $C^{\prime}$ depends only on $C$ and $q$.

Proof. Since $d_{j} \leq C \log (j+1) / j \leq C \sqrt{j}$, it follows that $\log \left(e+1 / d_{j}\right) \geq$ $C_{1} \log (j+1)$, where $C_{1}$ depends only on $C$. Therefore,

$$
\sum_{j=1}^{N} \phi\left(d_{j}\right) \leq C_{1}^{-q} \sum_{j=1}^{N} \frac{d_{j}}{(\log (j+1))^{q}}
$$

Next we use summation by parts, replacing $d_{j}$ with $s_{j}-s_{j-1}$, where $s_{0}=0$ 
by convention, to obtain

$$
\begin{aligned}
& \sum_{j=1}^{N} \frac{d_{j}}{(\log (j+1))^{q}} \\
& \quad=\frac{s_{N}}{(\log (N+1))^{q}}+\sum_{j=1}^{N-1} s_{j}\left(\frac{1}{(\log (j+1))^{q}}-\frac{1}{(\log (j+2))^{q}}\right)
\end{aligned}
$$

The first term on the right is bounded by $C /(\log (N+1))^{q-1}$. Since $s_{j} \leq$ $C \log (j+1)$ and

$$
\frac{1}{(\log (j+1))^{q}}-\frac{1}{(\log (j+2))^{q}} \leq \frac{q}{(j+1)(\log (j+1))^{1+q}},
$$

it follows that

$$
\begin{aligned}
& \sum_{j=1}^{N-1} s_{j}\left(\frac{1}{(\log (j+1))^{q}}-\frac{1}{(\log (j+2))^{q}}\right) \\
& \leq C \sum_{j=1}^{\infty} \frac{1}{(j+1)(\log (j+1))^{q}}=: C_{2}
\end{aligned}
$$

where $C_{2}$ depends only on $C$ and $q$. Combining (3.5)-(3.7), we obtain (3.4).

Proof of Theorem 3.1. Let $a, b \in \mathbb{R}^{n}$ be distinct. Given a partition $\left(a_{j}\right)_{j=0}^{N}$ of $[a, b]$, let $d_{1} \geq \cdots \geq d_{N}$ be the numbers $\left|f\left(a_{j}\right)-f\left(a_{j-1}\right)\right|$ arranged in nonincreasing order. By Lemma 3.3 the partial sums $s_{j}=d_{1}+\cdots+d_{j}$ are bounded by $C \log (2 j+2)|f(a)-f(b)|$, where $C$ is the constant in (3.2). Applying Lemma 3.4 we arrive at the conclusion of the theorem.

4. Failure of bounded variation: Proof of Theorem 1.5. Let $Q \subset \mathbb{C}$ be the open square $\{x+i y: 2<x<6,|y|<2\}$. The closure of $Q$ contains two smaller closed squares $Q_{1}=\{x+i y:|x-3|+|y| \leq 1\}$ and $Q_{2}=$ $\{x+i y:|x-5|+|y| \leq 1\}$.

Let $g: \bar{Q} \rightarrow \mathbb{C}$ be a Lipschitz function such that

$$
g(z)= \begin{cases}i(z-2), & z \in Q_{1}, \\ i(6-z), & z \in Q_{2}, \\ 0, & z \in \partial Q .\end{cases}
$$

Extend $g$ to the set $A=\bigcup_{k \in \mathbb{Z}}(\bar{Q}+8 k)$ so that $g(z+8)=g(z)$. Finally, set $g(z)=0$ for $z \notin A$.

Let $L$ be the Lipschitz constant of $g$. We shall prove that for $0<\epsilon<$ $1 /(2 L)$ the mapping

$$
f(z)=z+\epsilon \sum_{m=0}^{\infty} 4^{-m} g\left(4^{m} z\right), \quad z \in \mathbb{C},
$$




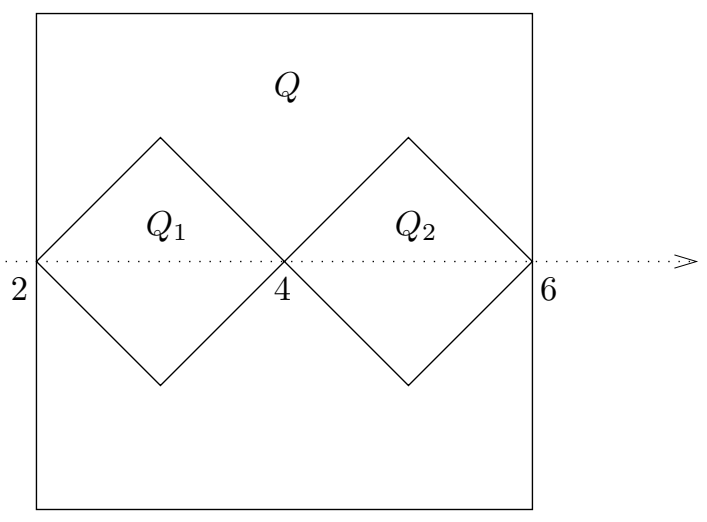

Fig. 1

satisfies

$$
\left|f_{\bar{z}}\right| \leq k \operatorname{Re} f_{z} \quad \text { a.e. } \quad \text { with } \quad k=\frac{\epsilon L}{1-\epsilon L} .
$$

First of all, the series in (4.1) converges uniformly because $g$ is bounded. Let

$$
B=\bigcup_{l \in \mathbb{Z}}\left\{\left[Q \backslash\left(Q_{1} \cup Q_{2}\right)\right]+8 l\right\}
$$

and note that

$$
B \subset \bigcup_{j \in \mathbb{Z}}\{z:|\operatorname{Re} z-2 j|<|\operatorname{Im} z|\}
$$

and

$$
B \cap \bigcup_{j \in \mathbb{Z}}\{z:|\operatorname{Re} z-8 j|<|\operatorname{Im} z|\}=\emptyset .
$$

We claim that for any $z \in \mathbb{C}$ there exists at most one integer $m \geq 0$ such that $4^{m} z \in B$. Indeed, let $m_{0}$ be the smallest such integer. Replacing $z$ with $4^{m_{0}} z$, we may assume that $m_{0}=0$, i.e., $z \in B$. According to (4.3), there exists $j \in \mathbb{Z}$ such that $|\operatorname{Re} z-2 j|<|\operatorname{Im} z|$. For any $m \geq 1$ the number $\zeta=4^{m} z$ satisfies $\left.\left|\operatorname{Re} \zeta-8 \cdot 4^{m-1} j\right|<|\operatorname{Im} \zeta|\right\}$, which implies $\zeta \notin B$ by (4.4). This proves the claim.

Since both $\operatorname{Re} g_{z}$ and $g_{\bar{z}}$ vanish a.e. outside of $B$, it follows that

$$
1-\epsilon L \leq \operatorname{Re} f_{z}(z) \leq 1+\epsilon L, \quad\left|f_{\bar{z}}(z)\right| \leq \epsilon L \quad \text { a.e. in } \mathbb{C} .
$$

This proves (4.2). Since $g(z)=0$ when $|\operatorname{Im} z|>2$, it follows that $\left|\operatorname{Im} f_{z}(z)\right| \leq$ $\epsilon m L$ when $|\operatorname{Im} z| \geq 2 \cdot 4^{-m}$. This implies

$$
|D f(z)| \leq C \log (e+1 /|\operatorname{Im} z|)
$$

for some constant $C$. Hence $f \in W_{\text {loc }}^{1, p}(\mathbb{C} ; \mathbb{C})$ for all $p<\infty$. Therefore, 
$f$ is quasiregular. By [13, Cor. 1.5] it is quasiconformal. It is clear that $\operatorname{Re} f(x)=x$ for all $x \in \mathbb{R}$.

It remains to prove that the function

$$
h(x):=\epsilon^{-1} \operatorname{Im} f(x)=-i \sum_{m=0}^{\infty} \frac{1}{4^{m}} g\left(4^{m} x\right), \quad x \in \mathbb{R},
$$

has infinite variation on any nontrivial interval $[a, b] \subset \mathbb{R}$. Due to the selfsimilar structure of $h$ it suffices to consider the interval $[0,8]$. We will show that the sum

$$
V_{N}:=\sum_{j=0}^{4^{N}}\left|h\left(\frac{8 j}{4^{N}}\right)-h\left(\frac{8 j-8}{4^{N}}\right)\right|
$$

satisfies

$$
V_{N} \geq c \sqrt{N}
$$

with an absolute constant $c>0$. Let $x_{j}=(8 j) 4^{-N}, j=0, \ldots, 4^{N}$. When $m \geq N$, we have $g\left(4^{m} x_{j}\right)=0$ for all $j$. Therefore, in the definition of $V_{N}$ we can replace $h$ with the partial sum

$$
h_{N}(x)=-i \sum_{m=0}^{N-1} \frac{1}{4^{m}} g\left(4^{m} x\right) .
$$

Since $h_{N}$ is affine on each interval $\left[x_{j-1}, x_{j}\right]$, it follows that $V_{N}=\int_{0}^{8}\left|h_{N}^{\prime}(x)\right| d x$. We claim that for a.e. $x \in \mathbb{R}$,

$$
\frac{d}{d x}(-i g(x))=\frac{1}{2}\left(s_{0}(x / 8)-s_{1}(x / 8)\right),
$$

where $s_{m}$ is the $m$ th Rademacher function, defined by

$$
s_{m}(x)=\operatorname{sign} \sin \left(2^{m+1} \pi x\right) .
$$

Since both sides of (4.7) are periodic functions with period 8, it suffices to check that equality holds a.e. on the interval $(0,8)$. From the definitions of $g$ and $s_{m}$ one can see that both sides of $(4.7)$ coincide with $\chi_{[2,4]}\left(-\chi_{[4,6]}\right)$ when $0<x<8$ and $x \neq 2,4,6$. This proves (4.7). It then follows that

$$
h_{N}^{\prime}(x)=\frac{1}{2} \sum_{m=0}^{N-1}\left(s_{2 m}(x / 8)-s_{2 m+1}(x / 8)\right) .
$$

The $L^{1}$ norm of a Rademacher series is comparable to the $\ell^{2}$ norm of its coefficients [25, Thm. V.8.4]. Hence $\int_{0}^{8}\left|h_{N}^{\prime}(x)\right| d x \geq c \sqrt{N}$ with an absolute constant $c>0$. This completes the proof of (4.6).

REMARK 4.1. The mapping $f$ constructed in the proof of Theorem 1.5 does not have finite $\phi$-variation on lines with $\phi$ as in (1.8) for $0 \leq q<1 / 2$. 
Proof. Using Jensen's inequality and the estimate (4.6), we obtain

$$
\begin{aligned}
\sum_{j=0}^{4^{N}} \phi\left(\left|h\left(x_{j}\right)-h\left(x_{j-1}\right)\right|\right) & \geq 4^{N} \phi\left(V_{N} / 4^{N}\right) \\
& \geq \frac{c \sqrt{N}}{\left(\log \left(e+4^{N} /(c \sqrt{N})\right)^{q}\right.} \rightarrow \infty
\end{aligned}
$$

as $N \rightarrow \infty$.

Proof of Corollary 1.6. We may assume that the lines $L_{j}$ are parallel to the the real axis; that is, $L_{j}=\left\{z \in \mathbb{C}: \operatorname{Im} z=b_{j}\right\}$ where $b_{j}$ are distinct real numbers. For $m=1,2, \ldots$ let $\epsilon_{m}=\min _{1 \leq j<\ell \leq m}\left|b_{j}-b_{\ell}\right|$ and choose $c_{m}>0$ so that

$$
c_{m}\left|b_{m}\right|<2^{-m}
$$

and

$$
c_{m} \log \left(e+1 / \epsilon_{m}\right)<2^{-m} .
$$

We define

$$
F(z)=\sum_{m=1}^{\infty} c_{m} f\left(z-i b_{m}\right)
$$

where $f$ is the mapping in (4.1). Note that $|f(z)| \leq|z|+M$ for some constant $M$. The sum in (4.10) converges locally uniformly because by (4.8)-(4.9),

$$
\left|c_{m} f\left(z-i b_{m}\right)\right| \leq c_{m}\left(|z|+\left|b_{m}\right|+M\right) \leq 2^{-m}(|z|+1+M) .
$$

By virtue of (4.2) the partial sums of the series (4.10) are $K$-quasiconformal with $K=(1+k) /(1-k)$. Since $F$ is a locally uniform limit of $K$-quasiconformal mappings, it is either constant or $K$-quasiconformal [17, II 5.3]. Below we show that $F$ has infinite variation on the lines $L_{j}$; in particular, $F$ is nonconstant.

We claim that for every $j=1,2, \ldots$ the sum

$$
R_{j}(z)=\sum_{m \neq j} c_{m} f\left(z-i b_{m}\right)
$$

is Lipschitz on the line $L_{j}$. By (4.5) the restriction of $f\left(z-i b_{m}\right)$ to $L_{j}$ is Lipschitz with a constant $C \log \left(e+1 /\left|b_{m}-b_{j}\right|\right)$. We estimate the Lipschitz constant of $R_{j}$ by

$$
\sum_{m \neq j} c_{m} \log \left(e+1 /\left|b_{m}-b_{j}\right|\right) \leq \sum_{m<j} c_{m} \log \left(e+1 / \epsilon_{j}\right)+\sum_{m>j} c_{m} \log \left(e+1 / \epsilon_{m}\right) .
$$

The first sum on the right has finitely many terms, and the second sum converges by (4.9). Since $F(z)=c_{j} f\left(z-i b_{j}\right)+R_{j}$, it follows that $F\left(L_{j}\right)$ is not locally rectifiable at any of its points. 
5. Reduced quasiconformal mappings in four dimensions. Our first goal in this section is to reformulate the definition of reduced quasiconformal mappings (Definition 1.4) in terms of differential matrices $D f(x) \in$ $\mathbb{M}_{n}$ rather than complex derivatives. This is done in Proposition 5.3 below. We write $\mathbb{M}_{n}$ for the set of all $n \times n$ matrices with real entries. Also, for $\delta \in[-1,1]$ we let

$$
\mathbb{M}_{n}(\delta)=\left\{A \in \mathbb{M}_{n}:\langle A v, v\rangle \geq \delta|A v||v| \text { for all } v \in \mathbb{R}^{n}\right\} .
$$

Proposition 5.1. For $\delta \in(0,1)$ let

$$
H(\delta)=\frac{1+\sqrt{1-\delta^{2}}}{1-\sqrt{1-\delta^{2}}}
$$

Then $\|A\|\left\|A^{-1}\right\| \leq H(\delta)$ for all nonzero matrices $A \in \mathbb{M}_{n}(\delta)$.

Proof. For $n=2$ this proposition was proved in [2, p. 84]. If $n \geq 3$, let $v$ and $w$ be distinct unit vectors such that $|A v|=\|A\|$ and $|A w|=\left\|A^{-1}\right\|^{-1}$. Applying the two-dimensional case to the subspace spanned by $v$ and $w$, we arrive at the desired conclusion.

A matrix $A \in \mathbb{M}_{2}$ determines a linear mapping $x \mapsto A x$ of the plane $\mathbb{R}^{2}$. The same linear mapping can be written as $z \mapsto \alpha^{+} z+\alpha^{-} \bar{z}$ for some $\alpha^{+}, \alpha^{-} \in \mathbb{C}$. The numbers $\alpha^{+}$and $\alpha^{-}$can be thought of as the conformal and anticonformal parts of $A$ (cf. [8]).

Proposition 5.2 ([2, Thm. 3.11.6]). A matrix A belongs to $\mathbb{M}_{2}(\delta)$ if and only if $\left|\alpha^{-}\right|+\delta\left|\operatorname{Im} \alpha^{+}\right| \leq \sqrt{1-\delta^{2}} \operatorname{Re} \alpha^{+}$.

A complex number $a+i b \in \mathbb{C}$ can be identified with the $2 \times 2$ matrix $Z=\left(\begin{array}{cc}a & -b \\ b & a\end{array}\right)$. Thus we may consider $\mathbb{C}$ as a linear subspace of $\mathbb{M}_{2}$. Within this subspace each matrix decomposes into real and imaginary parts: $\operatorname{Re} Z=\left(\begin{array}{ll}a & 0 \\ 0 & a\end{array}\right)$ and $\operatorname{Im} Z=\left(\begin{array}{cc}0 & -b \\ b & 0\end{array}\right)$. Given $A \in \mathbb{M}_{2}$, let $\mathbb{C}(A)$ be the orthogonal projection of $A$ onto the subspace $\mathbb{C}$.

Proposition 5.3. A mapping $f \in W_{\mathrm{loc}}^{1,2}\left(\mathbb{R}^{2} ; \mathbb{R}^{2}\right)$ is reduced quasiconformal in the sense of Definition 1.4 if and only if there exists $\delta>0$ such that for a.e. $x \in \mathbb{R}^{2}$ the derivative $A=D f(x)$ satisfies $A-\operatorname{Im} \mathbb{C}(A) \in \mathbb{M}_{2}(\delta)$.

Proof. Writing the matrix $A=D f(x)$ in conformal-anticonformal coordinates as $\left(\alpha^{+}, \alpha^{-}\right)$, we observe that $A-\operatorname{Im} \mathbb{C}(A)$ corresponds to $\left(\operatorname{Re} \alpha^{+}, \alpha^{-}\right)$. According to Proposition 5.2, the condition $A-\operatorname{Im} \mathbb{C}(A) \in \mathbb{M}_{2}(\delta)$ is equivalent to $\left|\alpha^{-}\right| \leq \sqrt{1-\delta^{2}} \operatorname{Re} \alpha^{+}$. The latter inequality is the same as (1.6) with $k=\sqrt{1-\delta^{2}}$. 
A quaternion $\alpha+\beta \mathbf{i}+\gamma \mathbf{j}+\zeta \mathbf{k}$ can be identified with a $4 \times 4$ real matrix

$$
Q=\left(\begin{array}{cccc}
\alpha & -\beta & -\gamma & -\zeta \\
\beta & \alpha & -\zeta & \gamma \\
\gamma & \zeta & \alpha & -\beta \\
\zeta & -\gamma & \beta & \alpha
\end{array}\right)
$$

With this identification we consider the set of quaternions $\mathbb{H}$ as a subset of $\mathbb{M}_{4}$. Since quaternion conjugation corresponds to matrix transposition, we have $Q^{T} Q=\|Q\|^{2} I$, where $\|Q\|$ is the operator norm of the matrix $Q$, also equal to the absolute value of the quaternion. Consequently, $|Q v|=\|Q\||v|$ for any vector $v \in \mathbb{R}^{4}$.

A quaternion $Q$ is the sum of its real (scalar) and imaginary parts:

$$
\operatorname{Re} Q=\left(\begin{array}{cccc}
\alpha & 0 & 0 & 0 \\
0 & \alpha & 0 & 0 \\
0 & 0 & \alpha & 0 \\
0 & 0 & 0 & \alpha
\end{array}\right), \quad \operatorname{Im} Q=Q-\operatorname{Re} Q .
$$

If $\operatorname{Re} Q=0$, the quaternion $Q$ is purely imaginary. For a matrix $A \in \mathbb{M}_{4}$, we define $\mathbb{H}(A)$ to be the orthogonal projection of $A$ onto the subspace $\mathbb{H} \subset \mathbb{M}_{4}$.

Definition 5.4. A homeomorphic mapping $f \in W_{\text {loc }}^{1,4}\left(\mathbb{R}^{4} ; \mathbb{R}^{4}\right)$ is reduced quasiconformal if there exists $\delta>0$ such that for a.e. $x \in \mathbb{R}^{4}$ the derivative $A=D f(x)$ satisfies $A-\operatorname{Im} \mathbb{H}(A) \in \mathbb{M}_{4}(\delta)$.

First of all, we need to justify the terminology by proving the following proposition.

Proposition 5.5. Any reduced quasiconformal mapping $f: \mathbb{R}^{4} \rightarrow \mathbb{R}^{4}$ is $K$-quasiconformal, where $K$ depends only on $\delta$ in Definition 5.4. In addition, $f$ is monotone.

Proof. The essence of this proposition is the algebraic implication

$$
A-\operatorname{Im} \mathbb{H}(A) \in \mathbb{M}_{4}(\delta) \Rightarrow\|A\|\left\|A^{-1}\right\| \leq \widetilde{H}(\delta) .
$$

We may assume that $A$ is a nonzero matrix. Let $Q=\operatorname{Im} \mathbb{H}(A)$ and $B=$ $A-Q$. If $Q=0$, then Proposition 5.1 gives (5.3) with $\widetilde{H}(\delta)=H(\delta)$. Assume $Q \neq 0$. Fix a unit vector $v \in \mathbb{R}^{4}$. Since $Q v /\|Q\|$ is a unit vector orthogonal to $v$, it follows that

$$
\langle B v, v\rangle^{2}+\|Q\|^{-2}\langle B v, Q v\rangle^{2} \leq|B v|^{2} .
$$

Using the inequality $\langle B v, v\rangle \geq \delta|B v|$, we obtain

$$
|\langle B v, Q v\rangle| \leq \sqrt{1-\delta^{2}}\|Q\||B v|
$$


which in turn yields

$$
\begin{aligned}
|A v|^{2} & =|B v+Q v|^{2}=|B v|^{2}+\|Q\|^{2}+2\langle B v, Q v\rangle \\
& \geq\left(1-\sqrt{1-\delta^{2}}\right)\left(|B v|^{2}+\|Q\|^{2}\right)+\sqrt{1-\delta^{2}}(|B v|-\|Q\|)^{2} \\
& \geq\left(1-\sqrt{1-\delta^{2}}\right)\left(|B v|^{2}+\|Q\|^{2}\right) .
\end{aligned}
$$

In particular, $A$ is invertible. We also have the trivial estimate

$$
|A v|^{2} \leq 2\left(|B v|^{2}+\|Q\|^{2}\right)
$$

Combining (5.5) and (5.6), we conclude that

$$
\begin{aligned}
\|A\|^{2}\left\|A^{-1}\right\|^{2} & =\frac{\max \left\{|A v|^{2}:|v|=1\right\}}{\min \left\{|A v|^{2}:|v|=1\right\}} \\
& \leq \frac{2}{1-\sqrt{1-\delta^{2}}} \frac{\max \left\{|B v|^{2}:|v|=1\right\}+\|Q\|^{2}}{\min \left\{|B v|^{2}:|v|=1\right\}+\|Q\|^{2}} \\
& \leq \frac{2}{1-\sqrt{1-\delta^{2}}} \frac{\max \left\{|B v|^{2}:|v|=1\right\}}{\min \left\{|B v|^{2}:|v|=1\right\}} \leq \frac{2\left(1+\sqrt{1-\delta^{2}}\right)^{2}}{\left(1-\sqrt{1-\delta^{2}}\right)^{3}}
\end{aligned}
$$

where the last step uses Proposition 5.1. This proves (5.3). Applying (5.3) to the derivative matrix $A=D f(x)$, we find that $f$ is $K$-quasiconformal with $K=\widetilde{H}(\delta)^{3}$.

With $A=D f(x)$ and $B=A-\operatorname{Im} \mathbb{H}(A)$ as above, we have

$$
\langle A v, v\rangle=\langle B v, v\rangle \geq 0, \quad v \in \mathbb{R}^{4} .
$$

Integrating this inequality along the segments $[a, b]$ on which $f$ is absolutely continuous, we obtain $\Delta_{f}(a, b) \geq 0$. The continuity of $f$ then implies $\Delta_{f}(a, b) \geq 0$ for all $a, b \in \mathbb{R}^{4}$.

REMARK 5.6. If in Definition 5.4 we do not require $f$ to be homeomorphic, then the proof of Proposition 5.5 shows that $f$ is $K$-quasiregular (see Definition 1.2).

It follows from Definition 5.4 that the set of reduced quasiconformal mappings is a convex cone in four dimensions as well as in two dimensions. Another similarity with the planar case is provided by the following result.

Proposition 5.7. Any nonconstant $\delta$-monotone mapping $f: \mathbb{R}^{4} \rightarrow \mathbb{R}^{4}$ is reduced quasiconformal in the sense of Definition 5.4.

Proof. Since $f$ is quasiconformal by $[15$, Cor. 7$]$, we have $f \in W_{\text {loc }}^{1,4}\left(\Omega ; \mathbb{R}^{4}\right)$. Fix a point $x \in \mathbb{R}^{4}$ where $f$ is differentiable and let $A=D f(x), Q=$ $\operatorname{Im} \mathbb{H}(A), B=A-Q$. The definition of $\delta$-monotonicity implies $A \in \mathbb{M}_{4}(\delta)$. For all $v \in \mathbb{R}^{n}$ we have $\langle Q v, v\rangle=0$ since $Q$ is an antisymmetric matrix. Thus,

$$
\langle B v, v\rangle=\langle A v, v\rangle \geq \delta|A v||v|, \quad v \in \mathbb{R}^{n} .
$$


It remains to prove that $|A v| \geq c|B v|$ for a constant $c>0$ that depends only on $\delta$. Note that $\operatorname{Im} \mathbb{H}(A)$ is the orthogonal projection of $A$ onto the space of purely imaginary quaternions, considered as a linear subspace of $\mathbb{M}_{4}$. Therefore, $B=A-\operatorname{Im} \mathbb{H}(A)$ is the projection of $A$ onto the orthogonal complement of the purely imaginary quaternions. Since orthogonal projections in $\mathbb{M}_{n}$ do not increase the Frobenius norm $\|\cdot\|_{F}$, it follows that

$$
\|B\| \leq\|B\|_{F} \leq\|A\|_{F} \leq 2\|A\|
$$

where we have used the relation between operator norm and Frobenius norm [12, p. 313]. Combining Proposition 5.1 with (5.8) we obtain

$$
|A v| \geq \frac{\|A\||v|}{H(\delta)} \geq \frac{\|B\||v|}{2 H(\delta)} \geq \frac{|B v|}{2 H(\delta)} .
$$

This estimate together with (5.7) imply $B \in \mathbb{M}_{4}(\delta / 2 H(\delta))$.

Our last result is an extension of Theorem 1.7 to four dimensions.

TheOREM 5.8. Let $f: \mathbb{R}^{4} \rightarrow \mathbb{R}^{4}$ be a reduced quasiconformal mapping in the sense of Definition 5.4. Then for any $q>1, f$ has finite $\phi$-variation on lines with $\phi$ as in (1.8).

Proof. For a purely imaginary quaternion $Q \in \mathbb{M}_{4}$ we define $f^{Q}(x)=$ $f(x)+Q x$. Recall the definition of the modulus of monotonicity $\Delta_{f}$ in (2.3). We claim that

$$
\Delta_{f}(a, b)=\min _{Q}\left|f^{Q}(a)-f^{Q}(b)\right|
$$

where the minimum is taken over all purely imaginary quaternions (and is attained). Indeed,

$$
\Delta_{f}(a, b)=\Delta_{f}(a, b) \leq\left|f^{Q}(a)-f^{Q}(b)\right| \quad \text { for any } Q \text { with } \operatorname{Re} Q=0 .
$$

In proving the converse inequality we may assume that $v:=a-b$ is a nonzero vector. Applying the unit quaternions $\mathbf{i}, \mathbf{j}$, and $\mathbf{k}$ to $v$, we obtain an orthogonal basis of $\mathbb{R}^{4}$, namely $\{v, \mathbf{i} v, \mathbf{j} v, \mathbf{k} v\}$. Expand the vector $f(a)-f(b)$ in this basis:

$$
f(a)-f(b)=\alpha v+\beta \mathbf{i} v+\gamma \mathbf{j} v+\zeta \mathbf{k} v .
$$

In these terms, $\Delta_{f}(a, b)=\langle\alpha v, v /|v|\rangle=\alpha|v|$. Since $f$ is monotone by Proposition 5.5, we have $\alpha \geq 0$. The quaternion $Q=-\beta \mathbf{i}-\gamma \mathbf{j}-\zeta \mathbf{k}$ satisfies

$$
\left|f^{Q}(a)-f^{Q}(b)\right|=\alpha|v|=\Delta_{f}(a, b),
$$

which proves (5.9). For future reference, observe that (5.10) implies that $|f(a)-f(b)| \geq|Q v|=\|Q\||v|$, hence

$$
\|Q\| \leq \frac{|f(a)-f(b)|}{|a-b|} .
$$


Since linear mappings trivially satisfy the conclusion of the theorem, we may assume that $f$ is nonlinear. By Remark 5.6 there exist $K<\infty$ such that $f^{Q}$ is $K$-quasiregular for all purely imaginary quaternions. Also, $f^{Q}$ is monotone by Proposition 5.5. By [16, Thm. 1.2] any monotone quasiregular mapping defined on $\mathbb{R}^{n}$ is either constant or a homeomorphism. Since $f$ is not linear, $f^{Q}$ cannot be constant. Thus $f^{Q}$ is $K$-quasiconformal. By [11, Thm. 11.14] the family $f^{Q}$ has a common modulus of quasisymmetry $\eta$. Given distinct points $a, b, c \in \mathbb{R}^{4}$, let $Q$ be a minimizing quaternion in (5.9). The quasisymmetry of $f^{Q}$ implies

$$
\left|f^{Q}(c)-f^{Q}(a)\right| \leq \eta\left(\frac{|c-a|}{|b-a|}\right)\left|f^{Q}(b)-f^{Q}(a)\right| .
$$

Here $\left|f^{Q}(b)-f^{Q}(a)\right|=\Delta_{f}(a, b)$. Using (5.11) we obtain

$$
\begin{aligned}
|f(c)-f(a)| & \leq|Q(c-a)|+\left|f^{Q}(c)-f^{Q}(a)\right| \\
& \leq \frac{|c-a|}{|a-b|}|f(a)-f(b)|+\eta\left(\frac{|c-a|}{|b-a|}\right) \Delta_{f}(a, b),
\end{aligned}
$$

which is (3.1). It remains to apply Theorem 3.1 to $f$.

Acknowledgements. We thank Tadeusz Iwaniec for many stimulating discussions on the subject of this paper. We are grateful to Pietro PoggiCorradini and to both anonymous referees for their valuable comments.

Research of L. V. Kovalev was supported by the NSF grant DMS-0913474. Research of J. Onninen was supported by the NSF grant DMS-0701059.

\section{References}

[1] G. Alessandrini and V. Nesi, Beltrami operators, non-symmetric elliptic equations and quantitative Jacobian bounds, Ann. Acad. Sci. Fenn. Math. 34 (2009), 47-67.

[2] K. Astala, T. Iwaniec, and G. J. Martin, Elliptic Partial Differential Equations and Quasiconformal Mappings in the Plane, Princeton Math. Ser. 48, Princeton Univ. Press, 2009.

[3] K. Astala and J. Jääskeläinen, Homeomorphic solutions to reduced Beltrami equations, Ann. Acad. Sci. Fenn. Math. 34 (2009), 607-613.

[4] Z. Balogh, P. Koskela, and S. Rogovin, Absolute continuity of quasiconformal mappings on curves, Geom. Funct. Anal. 17 (2007), 645-664.

[5] C. J. Bishop, Quasiconformal mappings which increase dimension, Ann. Acad. Sci. Fenn. Math. 24 (1999), 397-407.

[6] M. Bonk, J. Heinonen and E. Saksman, The quasiconformal Jacobian problem, in: In the Tradition of Ahlfors and Bers. III, Contemp. Math. 355, Amer. Math. Soc., Providence, RI, 2004, 77-96.

[7] L. C. Evans and R. F. Gariepy, Measure Theory and Fine Properties of Functions, CRC Press, Boca Raton, FL, 1992.

[8] D. Faraco and L. Székelyhidi, Tartar's conjecture and localization of the quasiconvex hull in $\mathbb{R}^{2 \times 2}$, Acta Math. 200 (2008), 279-305. 
[9] F. W. Gehring, The definitions and exceptional sets for quasiconformal mappings, Ann. Acad. Sci. Fenn. Ser. A I Math. 281 (1960), 1-28.

[10] - The $L^{p}$-integrability of the partial derivatives of a quasiconformal mapping, Acta Math. 130 (1973), 265-277.

[11] J. Heinonen, Lectures on Analysis on Metric Spaces, Universitext, Springer, New York, 2001.

[12] R. A. Horn and C. R. Johnson, Matrix Analysis, Cambridge Univ. Press, Cambridge, 1985.

[13] T. Iwaniec, L. V. Kovalev and J. Onninen, On injectivity of quasiregular mappings, Proc. Amer. Math. Soc. 137 (2009), 1783-1791.

[14] - - - 一, Dynamics of quasiconformal fields, preprint, arXiv:0811.4217.

[15] L. V. Kovalev, Quasiconformal geometry of monotone mappings, J. London Math. Soc. (2) 75 (2007), 391-408.

[16] L. V. Kovalev and J. Onninen, On invertibility of Sobolev mappings, preprint, arXiv:0812.2350.

[17] O. Lehto and K. I. Virtanen, Quasiconformal Mappings in the Plane, 2nd ed., Springer, New York, 1973.

[18] J. Malý, Absolutely continuous functions of several variables, J. Math. Anal. Appl. 231 (1999), 492-508.

[19] J. Musielak and W. Orlicz, On generalized variations. I, Studia Math. 18 (1959), $11-41$.

[20] Yu. G. Reshetnyak, Space Mappings with Bounded Distortion, Amer. Math. Soc., Providence, RI, 1989.

[21] S. Rickman, Quasiregular Mappings, Springer, Berlin, 1993.

[22] P. Tukia, A quasiconformal group not isomorphic to a Möbius group, Ann. Acad. Sci. Fenn. Ser. A I Math. 6 (1981), 149-160.

[23] D. Waterman, On convergence of Fourier series of functions of generalized bounded variation, Studia Math. 44 (1972), 107-117.

[24] L. C. Young, General inequalities for Stieltjes integrals and the convergence of Fourier series, Math. Ann. 115 (1938), 581-612.

[25] A. Zygmund, Trigonometric Series, 3rd ed., Cambridge Univ. Press, Cambridge, 2002 .

Department of Mathematics

Syracuse University

215 Carnegie

Syracuse, NY 13244, U.S.A.

E-mail: lvkovale@syr.edu jkonnine@syr.edu 